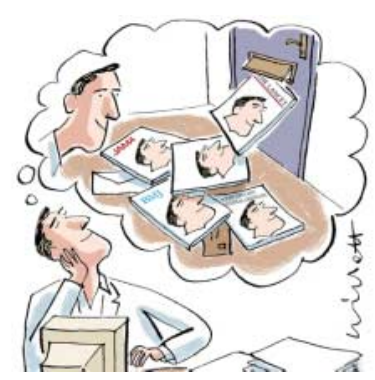

was about $76 \%$ of that of the United States; after their accession it fell to $66 \%$. In a bibliometric analysis, Soteriades and Falagas (p 192) compare research productivity of the EU, the four candidate countries (Bulgaria, Croatia, Romania, and Turkey) and the United States in several fields of biomedical sciences. The 10 newest member states and four candidate countries need further help and resources to increase their productivity, say the authors.

\title{
POEM* \\ Aspirin prevents stroke, but not MI, in women
}

Question Does aspirin prevent cardiovascular disease in women? Synopsis This study represents the largest and best evidence to date for women. Women over 45 without a history of coronary artery disease, cerebrovascular disease, or cancer were initially enrolled in a three month placebo run-in to establish compliance with the study protocol. Those who complied throughout the run-in period $(\mathrm{n}=39 \mathrm{876})$ were randomised (allocation not specified, but likely concealed) to receive either $100 \mathrm{mg}$ aspirin every other day or matching placebo. They were followed up for a mean of 10 years, with $97 \%$ complete data on morbidity and 99\% complete data on mortality. Very impressive. The mean age was 55 years, and the 10 year risk of heart disease was less than $5 \%$ in $85 \%$ of the women. Groups were balanced at the start of the study, outcomes were blindly assessed, and analysis was by intention to treat. Women taking aspirin were less likely to have a stroke $(1.1 \% v 1.3 \% ; \mathrm{P}=0.04$; number needed to treat $=444$ for 10 years $)$ or transient ischaemic attack $(0.9 \% v$ $1.2 \% ; \mathrm{P}=0.01 ; \mathrm{NNT}=384$ for 10 years) than women taking placebo. However, groups did not differ in the likelihood of myocardial infarction (MI) $(0.99 \%$ for aspirin and $0.97 \%$ for placebo) or death from cardiovascular causes $(0.60 \% v 0.63 \%)$, any major cardiovascular event $(2.4 \% v 2.6 \%)$, or any cause $(3.1 \%$ v 3.2\%). Gastrointestinal bleeds requiring transfusion were more common in the aspirin group $(0.64 \% v 0.46 \% ; \mathrm{P}=0.02$; number needed to treat to harm $=553$ for 10 years). The study was powered to have an $86 \%$ chance to detect a $25 \%$ reduction in the primary outcome of any major cardiovascular event. Review of the survival curve reveals a steady but small trend in favour of aspirin regarding the primary outcome. This apparent benefit, equivalent to a $5 \%$ to $10 \%$ relative reduction in all cause mortality, was not statistically significant despite the study's large size.

Bottom line Aspirin reduces the risk of stroke and transient ischaemic attack in women but does not reduce the risk of myocardial infarction or cardiovascular death. The reduction in strokes over 10 years (number needed to treat $=444$ ) must be balanced against an increase in serious gastrointestinal bleeds (number needed to treat to harm $=553$ ). No change in all cause mortality was seen in this large, long study.

Level of evidence $1 \mathrm{~b}$ (see www.infopoems.com/levels.html). Individual randomised controlled trials (with narrow confidence interval)

Ridker PM, Cook NR, Lee IM, et al. A randomized trial of low-dose aspirin in the primary prevention of cardiovascular disease in women. $N$ Engl J Med 2005;352:1293-304.

OinfoPOEMs 1992-2003 www.infoPOEMs.com/informationmastery.cfm * Patient-Oriented Evidence that Matters. See editorial (BMJ 2002;325:983)

\section{Editor's choice}

\section{Untangling a skein of wool}

Democracy is good for health-studies have shown this. But the transition from totalitarianism to democracy has taken its toll on the health of people in the former Soviet bloc. In almost all countries in the region, life expectancy has fallen in the past 10-15 years, as health systems struggled to cope with the loss of centralised bureaucracies and the rise of market forces. This theme issue is a snapshot of countries in transition, presenting honest appraisals of their shared problems and diverse attempts at solving them.

The challenges facing these countries are enormous. Economic collapse after transition has fuelled epidemics of HIV ( $p$ 216) and tuberculosis. Tobacco consumption is the single biggest cause of preventable death (pp 198, 191). Corruption is still endemic and healthcare infrastructure, especially in primary care, is lacking (pp 201, 204).

Many people thought that transition would allow them to keep the good things about the communist system-universal access to free health care-and lose the bad-lack of drugs, out of date training, failing technology, authoritarianism, and low wages. They have been disappointed. As Peter Toon says (p 243), "changing a health service is like untangling a skein of wool: pulling at one point creates a knot somewhere else. Health care cannot be reformed piecemeal." Reforms in Croatia have saved costs and increased efficiency but have created inequities and left Croatians feeling betrayed (pp 223, 226).

Miklós Szócska and colleagues describe the "perverted policy cycle" in Hungary (p 231). Managers in the newly emerging democracy were inexperienced. Health ministers changed constantly, each bringing a new set of ideas to meet the heightened expectations born of the latest political crisis. Reforms were introduced rapidly using law and regulation rather than training and communication, creating a damaging gap in perception: senior policy makers now believe that changes were effective, while managers feel they were frequent but superficial.

Amidst the challenges, Poland represents an important success story. Here coronary heart disease has fallen by over a third in the past 15 years, largely because of changes to the nation's diet ( $p$ 187). As Karen Lock and Martin McKee explain (p 188), these dietary changes have little to do with health promotion and almost all to do with economic reforms.

The clear message that emerges is one that all countries would do well to hear: improvements in health cannot be expected from health reform or promotion alone. Indeed, the real improvements in health have come from political and economic reform: in Poland (p 187), Croatia (p 208), Estonia (p 210), and the Slovak Republic (p 213). Meanwhile, intensive health promotion campaigns have failed to dent the growth in consumption of tobacco and alcohol, which still enjoy liberal tax status.

We can tell people what the healthy choices are, but unless we make it easy for them to make those choices, through sensible political and economic reform, we may cause only greater inequity.

Fiona Godlee editor (fgodlee@bmj.com) 